\title{
New Enhanced Artificial Bee Colony (JA-ABC5) Algorithm with Application for Reactive Power Optimization
}

\author{
Noorazliza Sulaiman, ${ }^{1}$ Junita Mohamad-Saleh, ${ }^{1}$ and Abdul Ghani Abro ${ }^{2}$ \\ ${ }^{1}$ School of Electrical \& Electronic Engineering, Universiti Sains Malaysia, 14300 Nibong Tebal, Penang, Malaysia \\ ${ }^{2}$ College of Engineering, King Saud University, Muzahmyiah Campus, Riyadh 11451, Saudi Arabia \\ Correspondence should be addressed to Junita Mohamad-Saleh; jms@usm.my
}

Received 24 June 2014; Accepted 6 October 2014

Academic Editor: Ahmad T. Azar

Copyright (C) 2015 Noorazliza Sulaiman et al. This is an open access article distributed under the Creative Commons Attribution License, which permits unrestricted use, distribution, and reproduction in any medium, provided the original work is properly cited.

\begin{abstract}
The standard artificial bee colony ( $\mathrm{ABC}$ ) algorithm involves exploration and exploitation processes which need to be balanced for enhanced performance. This paper proposes a new modified ABC algorithm named JA-ABC5 to enhance convergence speed and improve the ability to reach the global optimum by balancing exploration and exploitation processes. New stages have been proposed at the earlier stages of the algorithm to increase the exploitation process. Besides that, modified mutation equations have also been introduced in the employed and onlooker-bees phases to balance the two processes. The performance of JA-ABC5 has been analyzed on 27 commonly used benchmark functions and tested to optimize the reactive power optimization problem. The performance results have clearly shown that the newly proposed algorithm has outperformed other compared algorithms in terms of convergence speed and global optimum achievement.
\end{abstract}

\section{Introduction}

Bioinspired algorithms (BIAs) are metaheuristics method that imitates the biological phenomenon of nature $[1,2]$. Various BIAs have been developed to solve complex optimization problems. For example, Davidović et al. (2011) have implemented Bee Colony Optimization (BCO) algorithm to solve $p$-center problem [3] and, in 2012, Badar et al. have used particle swarm optimization (PSO) algorithm to handle a reactive power control problem [4]. Karaboga and Latifoglu then applied artificial bee colony $(\mathrm{ABC})$ algorithm as a tool to solve adaptive filtering noisy transcranial Doppler signal [5]. Bacanin and Tuba (2014) have recently employed firefly algorithm to encounter cardinality constrained mean-variance portfolio optimization problem [6]. A few new BIAs have also been developed such as in the work of Obagbuwa and Adewumi that introduced Improved Cockroach Swarm Optimization (CSO) algorithm. The algorithm includes the insertion of hunger element to the existing CSO to enhance the exploration capabilities and the diversity of cockroach population [7]. Meanwhile, Zhou et al. (2014) have proposed Cloud
Model Bat algorithm which is based on the ideas of bat echolocation together with the attribute of cloud model in order to depict good performance in optimization [8].

BIAs consist of several classes such as evolutionary algorithms (EA), swarm-intelligence-based (SI) algorithms, and many more. Among them, SI is the most prominent BIAs. SI algorithms imitate the social behavior of nature, such as bird flocking, fish schooling, and bees' swarming. SI has basically been a technique which is based on the interaction of organisms in a population, such as the flocks of bird and a swarm of bees. The optimization algorithms have been developed by observing the interaction among the swarm members [7]. Various optimization algorithms which are based on this technique have been successfully used in various optimization applications such as in real power loss minimization [4], estimation of induction motor's parameter [9], multilevel image thresholding [10], and many more. Among the techniques, optimization algorithms based on honeybees' behaviors have become the most commonly investigated and explored phenomenon by optimization researchers. Abbass 
(2001) has investigated marriage in honeybees [11]. Later on, Karaboga (2005) has proposed the artificial bee colony (ABC) algorithm based on the foraging behavior of honeybees [12]. Next, the concept of honeybees mating has been studied by Marinakis et al. [13] and Niknam et al. [14] in 2011. Besides that, the idea of the waggle dances of honeybees has been investigated by Duangphakdee et al. in 2011 who found out that the honeybees have complexity in waggle dances as soon as the sun comes close to its zenith. Thus, they have studied the relation of foraging and absconding to the azimuth [15].

ABC was proposed by Karaboga in 2005 [12]. It mimics the intelligent foraging behavior of honeybees that shows how organized the honeybees interact among them to search for food. $\mathrm{ABC}$ has fewer tuned parameters compared to other optimization algorithms such as genetic algorithm (GA) and differential evolution (DE). Thus, it is a simple and efficient optimization algorithm [16]. Moreover, $\mathrm{ABC}$ has been proven to show superior performance in comparison to other prominent optimization algorithms such as genetic algorithm (GA), differential evolution (DE), evolutionary strategies (ES), and particle swarm optimization (PSO) algorithms [16-18]. Nevertheless, $\mathrm{ABC}$ has been found to suffer from few limitations such as slow convergence speed $[19,20]$ and premature convergence $[21,22]$. Due to that, researchers have tried to solve them by developing various $\mathrm{ABC}$ variants, for example, Gbest-guided ABC (GABC) by Zhu and Kwong in 2010 [23], Best-so-far ABC (BsfABC) by Banharnsakun et al. [24], and Improved $\mathrm{ABC}$ (IABC) by Gao and Liu [25] in 2011 as well as modified ABC (MABC) by Gao and Liu [20], Global-best ABC (BABC) by Gao et al. [19], and enhanced ABC by Abro and Mohamad-Saleh [26] in 2012. However, some of these variants are still incapable of efficiently solving the problems, whilst a number of the variants could still be improved. For instance, the idea of IABC using the best solution is very convincing because it enhances the convergence speed [25]. Furthermore, its incorporation of random search equation into the algorithm is rather promising as the equation is known for its randomness and able to generate diverse population [25]. However, IABC is unable to solve Rosenbrock function as it is actually poor in exploitation [25]. Meanwhile, one of the $\mathrm{BABC}$ variants, $\mathrm{BABC}$, has also incorporated the idea of using the previous best solution as the guidance for the search [19]. With some adjustment to the solution search equation, $\mathrm{BABC} 1$ has shown the best performance among other variants at that time. Nevertheless, BABC1 is actually prone to premature convergence when dealing with complex multimodal problems [27]. With the motivation from one of the $\mathrm{BABC}$ variants which is $\mathrm{BABC} 2$, enhanced $\mathrm{ABC}$ (EABC) has been proposed with the idea to balance the exploration and exploitation abilities of the algorithm. Nonetheless, EABC has a tendency to suffer from slow convergence speed (i.e., lack of exploitation process) as shown in [28]. With the motivation from the existing $\mathrm{ABC}$ variants and their limitations, a new modified $\mathrm{ABC}$ is proposed in this paper. This new enhanced $\mathrm{ABC}$ is expected to give excellent performance in terms of convergence speed and robust global minimum search.

\section{Artificial Bee Colony (ABC) Algorithm Model}

The standard ABC algorithm is a population-based optimization algorithm. The working principle of $\mathrm{ABC}$ is as illustrated in Figure 1. Based on the figure, the working principle of $A B C$ can be categorized into five main phases which are initialization, employed-bees, onlooker-bees, scout-bee, and termination phases which consist of a total of twelve stages or processes.

In $\mathrm{ABC}$, three phases are performance-deciding phases which are employed-bees, onlooker-bees, and scout-bee phases while the other two are supporting phases. The exploration process of the algorithm takes place in employed-bees and onlooker-bees phases where the bees need to explore the neighborhood of the food sources allocated to them. Meanwhile, the exploitation process happens in the onlooker-bees phase when onlooker-bees apply fitness-proportion selection scheme in order to select the selected-fitter food sources. The details of the phases are discussed in the following subsections and more details of ABC can also be found in [18].

2.1. Initialization. In $\mathrm{ABC}$ algorithm, food sources represent the possible solution among the population of a problem. They are randomly initialized. The initialization of the population is based on user predetermined values of the population size. These food sources are then assigned to the employed-bees. Next, the nectar amounts which represent the fitness value of each food source are calculated using equation found in $[18,29,30]$ :

$$
\text { fit }_{i}= \begin{cases}\frac{1}{1+f_{i}}, & f_{i} \geq 0 \\ 1+\operatorname{abs}\left(f_{i}\right), & f_{i}<0\end{cases}
$$

where $f_{i}$ is objective function value of $i$ th food source.

2.2. Employed-Bees Phase. In this phase, employed-bees explore the neighborhood of the food sources assigned to them and update the food sources using the mutation equation given by

$$
z_{i j}=y_{i j}+\phi_{i j}\left(y_{i j}-y_{k j}\right)
$$

where $z_{i j}$ is the candidate solution of food sources, $y_{i j}$ is the $j$ th dimension of the $i$ th food sources, and $y_{k j}$ is the $k$ th food sources that are randomly chosen from a neighborhood of $i$ th food sources for $k \in[1,2, \ldots, S N]$ and $S N$ is the number of food sources. Subscripts $k$ and $i$ are mutually exclusive food sources. For the equation, $k$ and $j$ are chosen randomly and $j \in[1,2, \ldots, D]$ where $D$ represents the dimension of the search space and $\phi_{i j}$ is the control parameter that represents random number from $[-1,1]$, inclusively.

The explorations by employed-bees generate new food sources (i.e., candidate solutions of food sources). A selection between the candidate solution and the old food sources is based on which of them exhibits the best fitness value. This selection is done using greedy-selection scheme. The chosen food sources are potentially fitter food sources and are shared with onlooker-bees in onlooker-bees phase. 


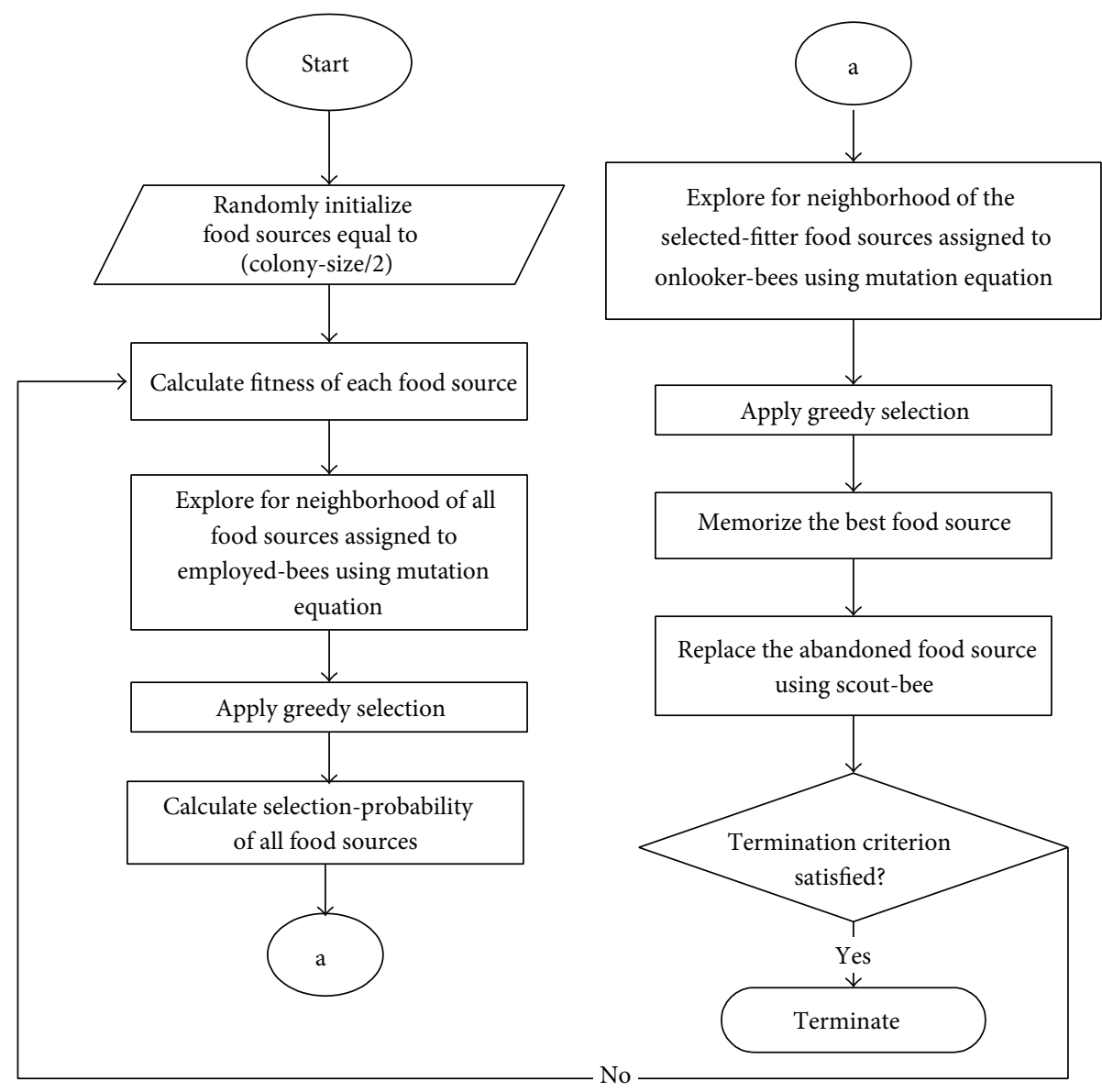

Figure 1: The flowchart of standard ABC algorithm.

2.3. Onlooker-Bees Phase. During this phase, the onlookerbees do not update all potentially fitter food sources shared with them by employed-bees. They apply fitness-proportion selection scheme to choose few selected-fitter food sources among all the food sources shared with them. The exploitation of the food sources by onlooker-bees has actually made the algorithm converge fast. The fitness-proportion selection scheme is dependent on the probability value, $P_{i}$ given by

$$
P_{i}=\frac{\text { fit }_{i}}{\sum_{j=1}^{S N} \mathrm{fit}_{j}},
$$

where $P_{i}$ is the probability of $i$ th food source, fit ${ }_{i}$ is the fitness value of $i$ th food source, and $S N$ represents the number of available food sources.

Onlooker-bees then explored the neighborhood of the selected-fitter food sources and update the food sources using the equation given in (2). The new candidate solution is then compared with the old food source using the greedy-selection scheme. Next, the best food source so far for that generation is memorized before entering the scout-bee phase.

2.4. Scout-Bee Phase. In scout-bee phase, a food source which has become exhausted and does not show improvement over a limit is abandoned [23]. Limit is a control parameter used to signify exhausted food source [19]. Employed-bee whose food source has reached limit will become scout-bee. The scout-bee will take consequent flights and search the search space randomly to find new food source using

$$
y_{i}^{j}=y_{\min }^{j}+\operatorname{rand}(0,1)\left(y_{\max }^{j}-y_{\min }^{j}\right),
$$

where $y_{\min }^{j}$ and $y_{\max }^{j}$ are the lower and upper limit of the search space, respectively. $\operatorname{rand}(0,1)$ is a function which randomly generates numbers within $[0,1]$. This action is necessary for the scout-bee to replace the abandoned food source with new food source and thus balance the number of populations again.

2.5. Termination. The termination criterion of the algorithm is based on the maximum number of generations or maximum cycle number (MCN) [18]. This number is preset by user prior to the simulation of $\mathrm{ABC}$ algorithm.

\section{New Enhanced ABC (JA-ABC5) Algorithm}

The limitations of $\mathrm{ABC}$ are due to (2) that is known to be good in exploration but poor in exploitation. This imbalances of exploration and exploitation capabilities of the standard $\mathrm{ABC}$ algorithm contribute to its lack in performance. Thus, 

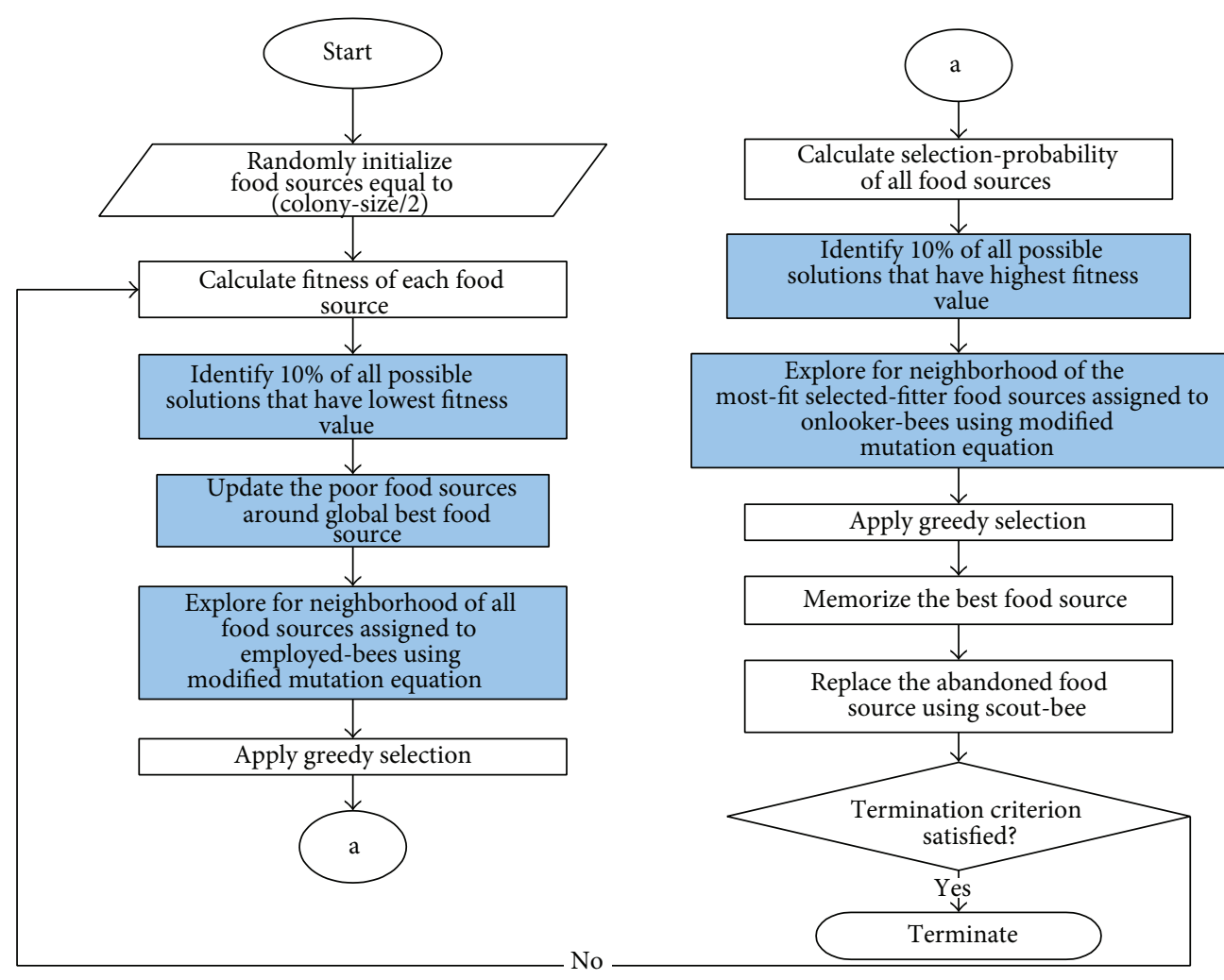

FIGURE 2: The flowchart of new enhanced ABC (JA-ABC5) algorithm.

few modifications have been introduced to the standard $\mathrm{ABC}$ algorithm for the purpose of balancing the exploration and exploitation capabilities of the algorithm. The proposed algorithm introduces four modifications to the standard $A B C$ algorithm as highlighted in Figure 2.

The first modification is the insertion of new phase between initialization and employed-bees phases. This phase consists of two stages illustrated by stages 4 and 5 in Figure 2 . The first stage aims to identify few food sources that have the lowest fitness values, referred to as poor food sources. Next, these poor food sources are updated around global best ( $g$-best) food source using the mutation equation inspired from [19] given by

$$
z_{i j}=y_{\text {best }, j}+\phi_{i j}\left(y_{p j}-y_{k j}\right),
$$

where $z_{i j}$ represents the candidate solution of $i$ th food source with $j$ th dimension. $y_{\text {best }, j}$ is the best food source, $y_{p j}$ is $j$ th dimension of $p$ th food source and is randomly chosen. Subscripts $i, k$, and $p$ are mutually exclusive food sources and the rest of the parameters are the same as in (2).

The generated food sources would now be fitter since they are being directed towards the global best food source based on (5). This has increased the exploitation process of the algorithm and makes the current population consist of fitter food sources. The random selection of food sources has also made the population not only fitter, but diverse as well.

Then, in employed-bees phases, the fitter populations are updated. Here comes the second modification which is represented by stage 6 in Figure 2. Since the population is now fitter, there is a possibility for the algorithm to be trapped in local optima. Thus, to overcome this, the exploration process should be enhanced. The enhancement of the exploration process has been done by adapting new mutation equation in employed-bees phase. This new mutation equation is obtained by adapting modified mutation equation inspired from [25] which is well known for its randomness. The modification produces a modified equation given by

$$
z_{i j}=y_{r 1 j}+\phi_{i j}\left(y_{r 2 j}-y_{r 3 j}\right),
$$

where $z_{i j}$ represents the candidate solution of $i$ th food source with $j$ th dimension. $y_{r 1 j}, y_{r 2 j}$, and $y_{r 3 j}$ are the $r 1$ th, $r 2$ th, and $r 3$ th food sources that are randomly chosen from neighborhood of $i$ th food sources. Subscripts $r 1, r 2$, and $r 3$ are mutually exclusive food sources and the rest of the parameters are the same as in (2). Equation (6) updates the food sources by directing the interaction among randomly chosen food sources. This increases the diversity of the exploration process that enhances the capability of the algorithm to avoid local optima trapping.

The next modification is aimed at increasing the convergence speed of the algorithm since random searching has a tendency to slow down the execution of the algorithm. The enhancement of the exploitation capability in onlookerbees phase has been formulated to overcome this problem. The onlooker-bees have been directed to update only few most-fit-selected-fitter food sources. As already mentioned, onlooker-bees basically do not update all food sources but update only selected-fitter food sources. Hence, in this 
proposed algorithm, onlooker-bees will update only few most-fit food sources among the selected-fitter food sources. Thus, with only few fitter food sources to be updated, the convergence speed of the algorithm has been increased. This modification is shown by stage 9 in Figure 2 .

The fourth modification is to replace the mutation of onlooker-bees from (2) to the equation adapted from the work of [25]

$$
z_{i j}=y_{\text {best }, j}+\phi_{i j}\left(y_{i j}-y_{m j}\right)
$$

where $z_{i j}$ represents the candidate solution of $i$ th food source with $j$ th dimension. $y_{\text {best }, j}$ is the best food source and $y_{m j}$ represents $j$ th dimension of $m$ th food source and is randomly chosen. Subscripts $i$ and $m$ are mutually exclusive food sources and the rest of the parameters are the same as (2).

Equation (7) is able to enhance the convergence speed since the fitter food sources in onlooker-bees phase have been updated towards the $g$-best food sources. This modification is presented by stage 10 in Figure 2. Thus, in the end, the proposed algorithm, JA-ABC5, has enhanced and balanced exploration and exploitation processes. With this, it is expected to converge faster and to be able to reach global optimum efficiently. Its ability is assessed by comparing its performance with existing variants on 27 benchmark functions and at solving the reactive power optimization problem.

\section{Simulations on Benchmark Functions}

In order to justify the robustness of the proposed JA-ABC5 algorithm, it has been simulated on 27 commonly used benchmark functions as listed in Table 1. These benchmark functions vary from different types of functions such as random shifted, unimodal, multimodal, and rotated functions prior to testing the capabilities of the algorithm to solve a wide range of problems.

The performance of JA-ABC5 has been compared with the standard $\mathrm{ABC}$ algorithm and three other sophisticated existing $A B C$ variants: Improved ABC (IABC) [25], Global best $\mathrm{ABC}$ (BABC1) [19], and enhanced ABC (EABC) [26, 29 ] to show the effectiveness of JA-ABC5 in solving those functions.

For all algorithms, the dimensionality of the benchmark functions has been set to 30 , the population size has been set to 50, number of generations has been limited to 1000, and the parameter limit has been set as $D \times S N$, where $D$ represents the dimension of the search space and $S N$ is the number of food sources. The $P$ value of IABC has been set to 0.25 [25]. As for global solution validation, each of the compared algorithms including JA-ABC5 has been set to be simulated for 30 times on each benchmark function [26]. All these values follow those used and recommended in the literature $[18-20,23,25,26,30]$.

The simulation and testing process have been carried out using Matlab R2010a on an Intel Core i7 with $2.80 \mathrm{GHz}$ speed computer.

4.1. Results of Benchmark Functions Simulation. Figures 3, $4,5,6$, and 7 show the graphical results of the proposed

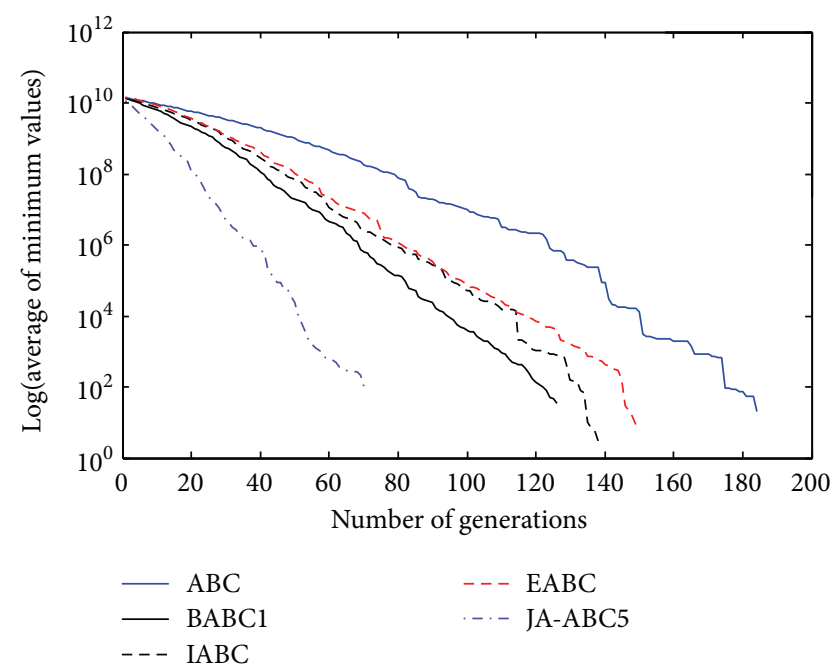

FIGURE 3: The convergence rates of optimization algorithms on Himmelblau function.

TABLE 1: Benchmark functions.

\begin{tabular}{|c|c|c|}
\hline Function & Function name & Initialization range \\
\hline$f 1$ & Griewank & \pm 600 \\
\hline$f 2$ & Rastrigin & \pm 15 \\
\hline$f 3$ & Rosenbrock & \pm 15 \\
\hline$f 4$ & RS Ackley & \pm 32 \\
\hline$f 5$ & Schwefel & \pm 500 \\
\hline$f 6$ & Himmelblau & \pm 600 \\
\hline$f 7$ & RS Sphere & \pm 600 \\
\hline$f 8$ & Step & \pm 600 \\
\hline$f 9$ & Bohachevsky 2 & \pm 100 \\
\hline$f 10$ & RS Schwefel 2.22 & \pm 100 \\
\hline$f 11$ & RS Schwefel Ridges & \pm 100 \\
\hline$f 12$ & RS Schwefel Ridges with Noise & \pm 15 \\
\hline$f 13$ & RS Elliptic & \pm 100 \\
\hline$f 14$ & Zekhelip & \pm 15 \\
\hline$f 15$ & Non-continuous Rastrigin & \pm 15 \\
\hline$f 16$ & Michalewicz & $0-180$ \\
\hline$f 17$ & First Expanded Function & \pm 15 \\
\hline$f 18$ & Second Expanded Function & \pm 15 \\
\hline$f 19$ & Third Expanded Function & \pm 15 \\
\hline$f 20$ & Fourth Expanded Function & \pm 500 \\
\hline$f 21$ & Fifth Expanded Function & \pm 100 \\
\hline$f 22$ & Sixth Expanded Function & \pm 100 \\
\hline$f 23$ & Seventh Expanded Function & \pm 15 \\
\hline$f 24$ & Eighth Expanded Function & \pm 100 \\
\hline$f 25$ & Rotated Griewank Function & $0-600$ \\
\hline$f 26$ & Rotated Ackley Function & \pm 32 \\
\hline$f 27$ & Rotated Rastrigin Function & \pm 5 \\
\hline
\end{tabular}

algorithm, JA-ABC5 algorithm. The figures have shown that the proposed algorithm has outperformed other algorithms in terms of convergence speed. It exhibits faster convergence 


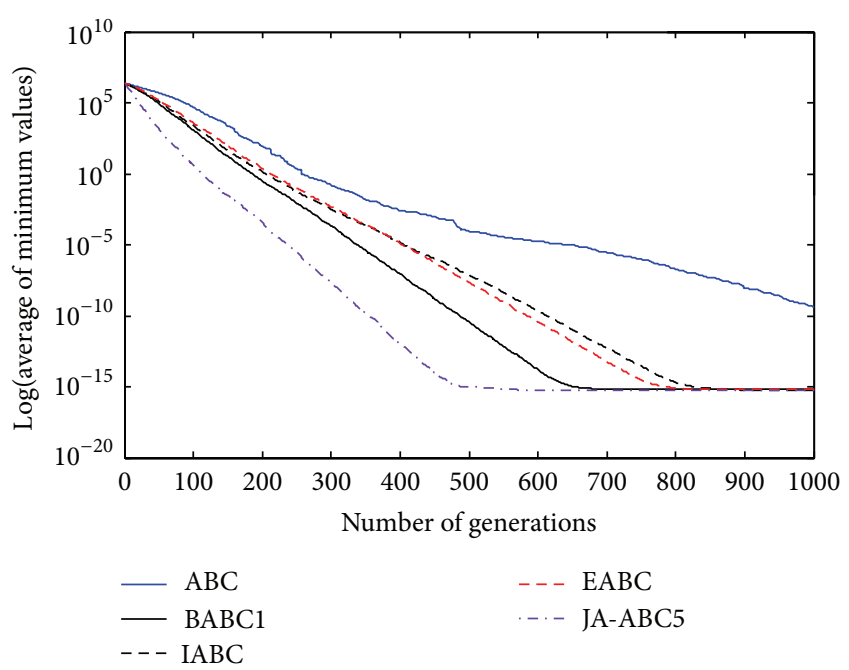

FIgURE 4: The convergence rates of optimization algorithms on Random Shifted Sphere function.

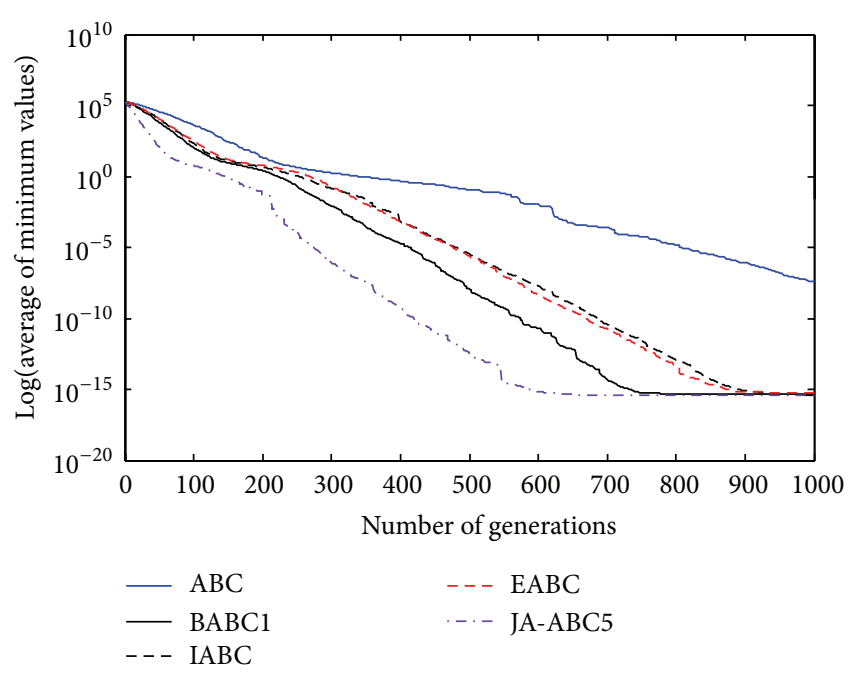

FIGURE 5: The convergence rates of optimization algorithms on Bohachevsky 2 function.

as compared to others. Moreover, the considerable difference of the proposed algorithm in comparison with other compared variants has clearly justified that the proposed algorithm is a robust $\mathrm{ABC}$ variant that has potential to solve optimization problems. The standard $\mathrm{ABC}$ exhibits the worst performance among all since it has suffered from few limitations as mentioned earlier.

Meanwhile, the statistical data in Table 2 reveal the numerical performance results of various $\mathrm{ABC}$ variants illustrating the values of minimum, mean, and standard deviation of the compared optimization algorithms. The results have shown that JA-ABC5 exhibits the least value of minimum, mean, and standard deviation on most of the benchmark functions. Thus, this vividly demonstrates that JA-ABC5 has the best performance in comparison with other compared $\mathrm{ABC}$ variants.

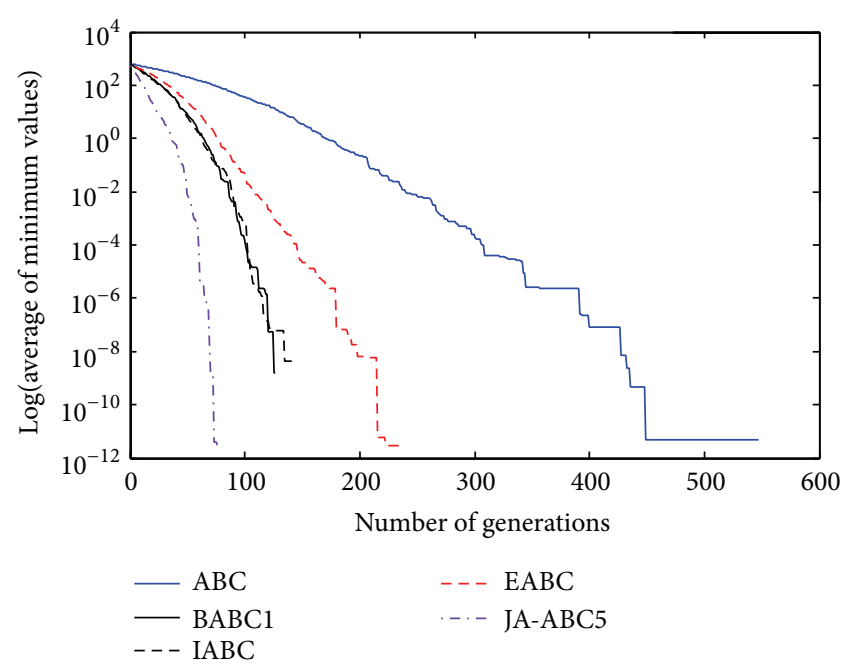

FIgURE 6: The convergence rates of the optimization algorithms on Rotated Griewank function.

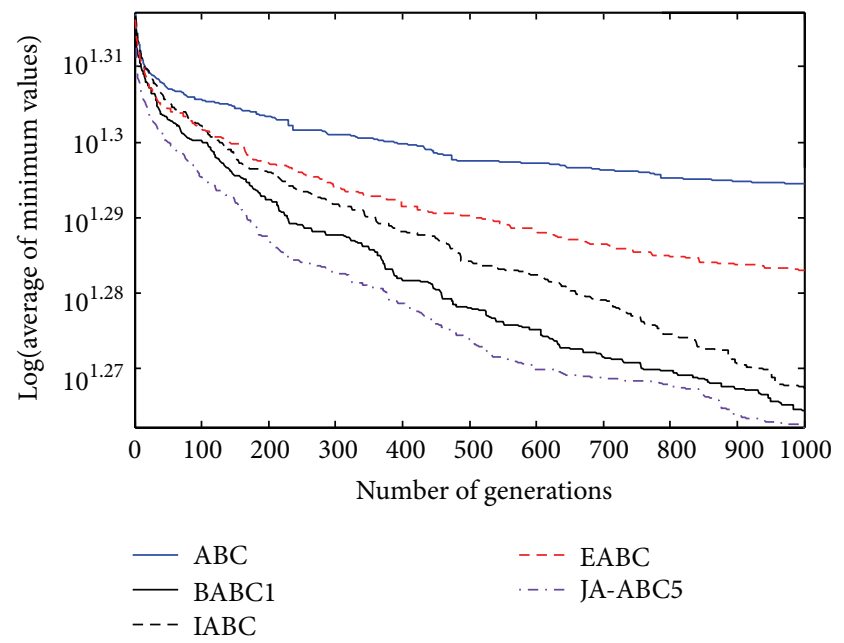

FIGURE 7: The convergence rates of the optimization algorithms on Rotated Ackley function.

\section{Reactive Power Optimization Application}

Reactive power optimization (RPO) is known to be a largescale nonlinear combinatorial constrained problem [31]. RPO basically serves to determine the optimal setting of the power system network to satisfy few constraints such as the power flow equation system security and equipment operating limits [32]. This problem has been discovered by Carpentier in 1962 [33] and, since then, many have tried to solve it. Researchers and engineers have tried to solve it by developing various search strategies since this kind of problem is very essential to be solved. This is because this problem is the important tool in the power system's operation and planning [34] since it actually has close contact with the security and economic dispatch of a power system [35]. For example, they have attempted to solve RPO problem using various classical methods such 
TABLE 2: Statistical Results of Optimization Algorithms.

\begin{tabular}{|c|c|c|c|c|c|c|c|}
\hline$f 1$ & MIN & Average & STD DEV & $f 15$ & MIN & Average & STD DEV \\
\hline $\mathrm{ABC}$ & $1.22 E-14$ & $1.47 E-13$ & $1.65 E-13$ & $\mathrm{ABC}$ & $3.56 E-07$ & $9.44 E-01$ & $8.38 E-01$ \\
\hline $\mathrm{BABC1}$ & $4.44 E-16$ & $6.22 E-16$ & $1.50 E-16$ & $\mathrm{BABC1}$ & $0.00 E+00$ & $2.00 E-01$ & $4.84 E-01$ \\
\hline IABC & $4.44 E-16$ & $6.40 E-16$ & $4.82 E-16$ & IABC & $0.00 E+00$ & $7.01 E-14$ & $6.95 E-14$ \\
\hline $\mathrm{EABC}$ & $4.44 E-16$ & $6.31 E-16$ & $1.58 E-16$ & $\mathrm{EABC}$ & $0.00 E+00$ & $6.72 E-12$ & $2.10 E-11$ \\
\hline JA-ABC5 & $3.33 E-16$ & $5.48 E-16$ & $8.71 E-17$ & JA-ABC5 & $0.00 E+00$ & $1.00 E-01$ & $3.05 E-01$ \\
\hline$f 2$ & MIN & Average & STD DEV & $f 16$ & MIN & Average & STD DEV \\
\hline $\mathrm{ABC}$ & $2.29 E-08$ & $3.77 E-01$ & $5.87 E-01$ & $\mathrm{ABC}$ & $-2.92 E+01$ & $-2.90 E+01$ & $1.40 E-01$ \\
\hline $\mathrm{BABC} 1$ & $0.00 E+00$ & $2.98 E-01$ & $4.64 E-01$ & $\mathrm{BABC} 1$ & $-2.96 E+01$ & $-2.94 E+01$ & $1.08 E-01$ \\
\hline IABC & $0.00 E+00$ & $0.00 E+00$ & $0.00 E+00$ & IABC & $-2.96 E+01$ & $-2.96 E+01$ & $1.58 E-02$ \\
\hline $\mathrm{EABC}$ & $0.00 E+00$ & $8.02 E-14$ & $3.02 E-13$ & EABC & $-2.96 E+01$ & $-2.95 E+01$ & $9.68 E-02$ \\
\hline JA-ABC5 & $0.00 E+00$ & $2.32 E-01$ & $5.01 E-01$ & JA-ABC5 & $-2.96 E+01$ & $-2.90 E+01$ & $1.29 E-02$ \\
\hline$f 3$ & MIN & Average & STD DEV & $f 17$ & MIN & Average & STD DEV \\
\hline $\mathrm{ABC}$ & $2.23 E-02$ & $9.03 E+00$ & $2.11 E+00$ & $\mathrm{ABC}$ & $7.85 E-03$ & $4.09 E-02$ & $1.81 E-01$ \\
\hline BABC1 & $2.32 E-02$ & $3.04 E+01$ & $3.30 E+01$ & BABC1 & $7.85 E-03$ & $5.69 E-01$ & $5.62 E-01$ \\
\hline $\mathrm{IABC}$ & $9.66 E-02$ & $5.63 E+00$ & $5.85 E+00$ & IABC & $7.85 E-03$ & $4.08 E-02$ & $1.81 E-01$ \\
\hline $\mathrm{EABC}$ & $1.48 E-02$ & $4.42 E+00$ & $7.16 E+00$ & $\mathrm{EABC}$ & $7.85 E-03$ & $7.85 E-03$ & $1.75 E-16$ \\
\hline JA-ABC5 & $1.31 E-02$ & $4.97 E+00$ & $1.44 E+01$ & JA-ABC5 & $3.03 E-03$ & $6.55 E-03$ & $1.37 E-01$ \\
\hline$f 4$ & MIN & Average & STD DEV & $f 18$ & MIN & Average & STD DEV \\
\hline $\mathrm{ABC}$ & $2.48 E-06$ & $1.82 E-05$ & $1.12 E-05$ & $\mathrm{ABC}$ & $1.59 E-14$ & $3.29 E-13$ & $2.53 E-13$ \\
\hline $\mathrm{BABC} 1$ & $3.46 E-14$ & $4.86 E-14$ & $7.52 E-15$ & $\mathrm{BABC1}$ & $4.42 E-16$ & $6.29 E-16$ & $1.05 E-16$ \\
\hline IABC & $8.98 E-12$ & $1.97 E-11$ & $6.75 E-12$ & IABC & $4.44 E-16$ & $5.72 E-16$ & $8.91 E-17$ \\
\hline EABC & $1.66 E-12$ & $4.15 E-12$ & $1.56 E-12$ & EABC & $4.92 E-16$ & $6.62 E-16$ & $1.23 E-16$ \\
\hline JA-ABC5 & $2.75 E-14$ & $3.12 E-14$ & $3.16 E-15$ & JA-ABC5 & $4.19 E-16$ & $5.32 E-16$ & $7.03 E-17$ \\
\hline$f 5$ & MIN & Average & STD DEV & $f 19$ & MIN & Average & STD DEV \\
\hline $\mathrm{ABC}$ & $1.07 E-01$ & $2.96 E+02$ & $1.20 E+02$ & $\mathrm{ABC}$ & $2.80 E+01$ & $3.13 E+01$ & $6.05 E+00$ \\
\hline $\mathrm{BABC1}$ & $3.82 E-04$ & $1.54 E+02$ & $1.33 E+02$ & $\mathrm{BABC1}$ & $2.78 E+01$ & $6.29 E+01$ & $3.13 E+01$ \\
\hline IABC & $3.82 E-04$ & $6.71 E+01$ & $8.62 E+01$ & IABC & $2.80 E+01$ & $4.20 E+01$ & $1.87 E+01$ \\
\hline EABC & $3.82 E-04$ & $9.28 E+01$ & $1.06 E+02$ & EABC & $2.76 E+01$ & $4.76 E+01$ & $2.68 E+01$ \\
\hline JA-ABC5 & $3.82 E-04$ & $1.03 E+02$ & $1.23 E+02$ & JA-ABC5 & $2.74 E+01$ & $4.18 E+01$ & $1.30 E+01$ \\
\hline$f 6$ & MIN & Average & STD DEV & $f 20$ & MIN & Average & STD DEV \\
\hline $\mathrm{ABC}$ & $-7.83 E+01$ & $-7.83 E+01$ & $1.55 E-07$ & $\mathrm{ABC}$ & $3.20 E-08$ & $6.65 E-02$ & $3.64 E-01$ \\
\hline $\mathrm{BABC1}$ & $-7.83 E+01$ & $-7.82 E+01$ & $4.09 E-01$ & BABC1 & $0.00 E+00$ & $5.98 E-01$ & $9.30 E-01$ \\
\hline IABC & $-7.83 E+01$ & $-7.83 E+01$ & $1.21 E-14$ & IABC & $0.00 E+00$ & $0.00 E+00$ & $0.00 E+00$ \\
\hline EABC & $-7.83 E+01$ & $-7.83 E+01$ & $2.28 E-01$ & $\mathrm{EABC}$ & $0.00 E+00$ & $1.67 E-16$ & $5.18 E-16$ \\
\hline JA-ABC5 & $-7.83 E+01$ & $-7.83 E+01$ & $2.39 E-01$ & JA-ABC5 & $0.00 E+00$ & $1.33 E-01$ & $5.06 E-01$ \\
\hline$f 7$ & MIN & Average & STD DEV & $f 21$ & MIN & Average & STD DEV \\
\hline $\mathrm{ABC}$ & $4.60 E-11$ & $4.53 E-10$ & $3.99 E-10$ & $\mathrm{ABC}$ & $1.66 E-10$ & $2.30 E-09$ & $3.28 E-09$ \\
\hline BABC1 & $4.55 E-16$ & $6.66 E-16$ & $1.10 E-16$ & BABC1 & $1.11 E-16$ & $3.89 E-16$ & $1.66 E-16$ \\
\hline IABC & $4.09 E-16$ & $5.89 E-16$ & $9.03 E-16$ & IABC & $5.55 E-17$ & $3.11 E-16$ & $1.85 E-16$ \\
\hline EABC & $4.72 E-16$ & $6.57 E-16$ & $1.24 E-16$ & EABC & $1.11 E-16$ & $4.86 E-16$ & $1.32 E-16$ \\
\hline JA-ABC5 & $3.29 E-16$ & $5.62 E-16$ & $1.08 E-16$ & JA-ABC5 & $5.55 E-17$ & $3.05 E-16$ & $1.21 E-16$ \\
\hline$f 8$ & MIN & Average & STD DEV & $f 22$ & MIN & Average & STD DEV \\
\hline $\mathrm{ABC}$ & $4.27 E-11$ & $4.82 E-10$ & $5.12 E-10$ & $\mathrm{ABC}$ & $1.80 E-10$ & $2.89 E-09$ & $3.60 E-09$ \\
\hline $\mathrm{BABC1}$ & $4.54 E-16$ & $6.37 E-16$ & $1.02 E-16$ & BABC1 & $0.00 E+00$ & $3.94 E-16$ & $1.45 E-16$ \\
\hline IABC & $2.87 E-16$ & $5.38 E-16$ & $9.62 E-17$ & IABC & $0.00 E+00$ & $2.55 E-16$ & $1.30 E-16$ \\
\hline EABC & $4.69 E-16$ & $6.65 E-16$ & $8.62 E-17$ & EABC & $2.22 E-16$ & $4.37 E-16$ & $1.33 E-16$ \\
\hline JA-ABC5 & $4.21 E-16$ & $4.66 E-16$ & $7.66 E-17$ & JA-ABC5 & $0.00 E+00$ & $2.46 E-16$ & $1.28 E-16$ \\
\hline
\end{tabular}


TABle 2: Continued.

\begin{tabular}{|c|c|c|c|c|c|c|c|}
\hline$f 9$ & MIN & Average & STD DEV & $f 23$ & MIN & Average & STD DEV \\
\hline $\mathrm{ABC}$ & $9.72 E-10$ & $3.85 E-08$ & $4.32 E-08$ & $\mathrm{ABC}$ & $7.05 E-12$ & $1.60 E-10$ & $1.70 E-10$ \\
\hline $\mathrm{BABC1}$ & $1.11 E-16$ & $4.57 E-16$ & $1.36 E-16$ & $\mathrm{BABC1}$ & $4.32 E-16$ & $6.20 E-16$ & $1.07 E-16$ \\
\hline IABC & $1.11 E-16$ & $4.09 E-16$ & $1.42 E-16$ & IABC & $3.23 E-16$ & $5.57 E-16$ & $8.87 E-17$ \\
\hline $\mathrm{EABC}$ & $2.22 E-16$ & $5.41 E-16$ & $1.76 E-16$ & EABC & $4.80 E-16$ & $6.78 E-16$ & $9.50 E-17$ \\
\hline JA-ABC5 & $1.11 E-16$ & $3.81 E-16$ & $1.27 E-16$ & JA-ABC5 & $3.22 E-16$ & $5.57 E-16$ & $8.72 E-17$ \\
\hline$f 10$ & MIN & Average & STD DEV & $f 24$ & MIN & Average & STD DEV \\
\hline $\mathrm{ABC}$ & $1.96 E-06$ & $1.07 E-05$ & $5.44 E-06$ & $\mathrm{ABC}$ & $7.50 E-01$ & $7.50 E-01$ & $2.20 E-04$ \\
\hline $\mathrm{BABC1}$ & $5.55 E-17$ & $2.44 E-15$ & $1.79 E-15$ & $\mathrm{BABC1}$ & $7.50 E-01$ & $7.50 E-01$ & $3.23 E-16$ \\
\hline IABC & $6.93 E-12$ & $2.64 E-11$ & $9.63 E-12$ & IABC & $7.50 E-01$ & $7.50 E-01$ & $3.44 E-16$ \\
\hline EABC & $2.95 E-12$ & $8.20 E-12$ & $4.41 E-12$ & EABC & $7.50 E-01$ & $7.50 E-01$ & $1.78 E-16$ \\
\hline JA-ABC5 & $0.00 E+00$ & $1.85 E-18$ & $1.01 E-17$ & JA-ABC5 & $7.50 E-01$ & $7.50 E-01$ & $1.36 E-16$ \\
\hline$f 11$ & MIN & Average & STD DEV & $f 25$ & MIN & Average & STD DEV \\
\hline $\mathrm{ABC}$ & $1.85 E-10$ & $2.62 E-09$ & $2.40 E-09$ & $\mathrm{ABC}$ & $0.00 E+00$ & $0.00 E+00$ & $0.00 E+00$ \\
\hline $\mathrm{BABC1}$ & $3.93 E-16$ & $6.42 E-16$ & $1.11 E-16$ & BABC1 & $0.00 E+00$ & $0.00 E+00$ & $0.00 E+00$ \\
\hline $\mathrm{IABC}$ & $3.95 E-16$ & $5.77 E-16$ & $9.15 E-17$ & IABC & $0.00 E+00$ & $0.00 E+00$ & $0.00 E+00$ \\
\hline EABC & $4.39 E-16$ & $6.54 E-16$ & $1.04 E-16$ & EABC & $0.00 E+00$ & $0.00 E+00$ & $0.00 E+00$ \\
\hline JA-ABC5 & $3.31 E-16$ & $5.76 E-16$ & $1.06 E-17$ & JA-ABC5 & $0.00 E+00$ & $0.00 E+00$ & $0.00 E+00$ \\
\hline$f 12$ & MIN & Average & STD DEV & $f 26$ & MIN & Average & STD DEV \\
\hline $\mathrm{ABC}$ & $3.33 E-03$ & $8.34 E-03$ & $3.92 E-03$ & $\mathrm{ABC}$ & $1.79 E+01$ & $1.97 E+01$ & $4.65 E-01$ \\
\hline $\mathrm{BABC1}$ & $4.60 E-13$ & $1.77 E-12$ & $1.30 E-12$ & $\mathrm{BABC1}$ & $1.70 E+01$ & $1.82 E+01$ & $6.89 E-01$ \\
\hline IABC & $5.65 E-09$ & $1.82 E-08$ & $1.15 E-08$ & IABC & $1.66 E+01$ & $1.85 E+01$ & $7.64 E-01$ \\
\hline $\mathrm{EABC}$ & $2.50 E-10$ & $8.02 E-10$ & $5.34 E-10$ & $\mathrm{EABC}$ & $1.80 E+01$ & $1.92 E+01$ & $5.92 E-01$ \\
\hline JA-ABC5 & $5.24 E-16$ & $8.08 E-16$ & $1.71 E-16$ & JA-ABC5 & $1.60 E+01$ & $1.73 E+01$ & $3.86 E-01$ \\
\hline$f 13$ & MIN & Average & STD DEV & $f 27$ & MIN & Average & STD DEV \\
\hline $\mathrm{ABC}$ & $1.63 E-10$ & $7.50 E-09$ & $1.60 E-08$ & $\mathrm{ABC}$ & $2.98 E+02$ & $3.25 E+02$ & $1.36 E+01$ \\
\hline $\mathrm{BABC1}$ & $4.49 E-16$ & $6.38 E-16$ & $1.06 E-16$ & $\mathrm{BABC} 1$ & $2.12 E+02$ & $2.88 E+02$ & $2.01 E+01$ \\
\hline $\mathrm{IABC}$ & $4.21 E-16$ & $5.41 E-16$ & $9.08 E-17$ & IABC & $2.59 E+02$ & $2.99 E+02$ & $1.97 E+01$ \\
\hline $\mathrm{EABC}$ & $4.64 E-16$ & $6.34 E-16$ & $1.06 E-16$ & EABC & $2.50 E+02$ & $2.93 E+02$ & $2.32 E+01$ \\
\hline JA-ABC5 & $2.67 E-16$ & $5.30 E-16$ & $8.66 E-17$ & JA-ABC5 & $2.08 E+02$ & $2.85 E+02$ & $1.26 E+01$ \\
\hline$f 14$ & MIN & Average & STD DEV & & & & \\
\hline $\mathrm{ABC}$ & $3.32 E-08$ & $4.03 E-04$ & $1.31 E-03$ & & & & \\
\hline $\mathrm{BABC1}$ & $4.08 E-16$ & $7.40 E-03$ & $2.82 E-02$ & & & & \\
\hline IABC & $2.72 E-16$ & $1.06 E-15$ & $2.41 E-15$ & & & & \\
\hline $\mathrm{EABC}$ & $3.03 E-16$ & $1.43 E-11$ & $4.70 E-11$ & & & & \\
\hline JA-ABC5 & $2.57 E-16$ & $3.70 E-03$ & $2.03 E-02$ & & & & \\
\hline
\end{tabular}

as linear programming, Newton method, interior point, and many more. Nonetheless, the methods have shown some inefficiency in solving it [31]. Recently, researchers have tried to implement stochastic and heuristics techniques to solve this problem [31]. Thus, this has shown that RPO basically can be a perfect tool in order to validate the robustness of the proposed algorithm.

RPO problem is a combinatorial nonlinear constrained problem. The general mathematical formulation for that kind of problem is given by

$$
\min (f(x))
$$

such that

$$
\begin{aligned}
& g(x)=0, \\
& h(x) \leq 0,
\end{aligned}
$$

where $f(x)$ is the objective function to be minimized, $g(x)$ is the equality constraints, and $h(x)$ is the inequality constraints. Hence, the mathematical formulation of RPO problem with equality and inequality constraints is discussed in next subsections.

5.1. Objective Function-Active Power Loss. The objective function for RPO problem can be either the active power loss, total cost of compensation, total energy generation cost, and many 
more [31]. In this paper, only active power loss is considered as the objective function to be solved by the proposed algorithm, JA-ABC5. The mathematical formulation of active power loss is given by

$$
\begin{aligned}
P_{\text {loss }} & =\sum_{k=1}^{N L} P_{\text {loss }, k} \\
& =\sum_{i=1}^{N} \sum_{j=1}^{N} g_{i j}\left[V_{i}^{2}+V_{j}^{2}-2 V_{i} V_{j} \cos \theta_{i j}\right],
\end{aligned}
$$

where $P_{\text {loss }}$ is an active power loss, $g_{i j}$ is the conductance between bus $i$ and bus $j, V_{i}$ is the voltage magnitude of bus $i$, $V_{j}$ is the voltage magnitude of bus $j, \theta_{i j}$ is the angle difference of $i j$ th transmission line, $N$ is the total number of system's buses, and $N L$ is the total number of transmission lines.

5.2. Equality Constraints. The equality constraints of the problem has been set to the power flow equations given by [36]

$$
\begin{gathered}
P_{G i}-P_{D i}-\sum_{j=1}^{N}\left|V_{i}\right|\left|V_{j}\right|\left|Y_{i j}\right| \cos \left(\theta_{i j}-\delta_{i}+\delta_{j}\right)=0, \\
Q_{G i}-Q_{D i}+\sum_{j=1}^{N}\left|V_{i}\right|\left|V_{j}\right|\left|Y_{i j}\right| \sin \left(\theta_{i j}-\delta_{i}+\delta_{j}\right)=0,
\end{gathered}
$$

where $P_{G i}$ is the active power generation at bus $i, P_{D i}$ is the active power demand at bus $i, Q_{G i}$ is the reactive power generation at bus $i, Q_{D i}$ is the reactive power demand at bus $i$, $Y_{i j}$ is the admittance between bus $i$ and bus $j, \delta i$ and $\delta j$ are the voltage angle at bus $i$ and bus $j$, respectively, and the rest of the parameters are the same as in (10).

5.3. Inequality Constraints. The inequality constraints of the problem are the control variables that are to be optimized within their ranges. These control variables are the food sources or possible solutions that need to be optimized by JAABC5. The range of the possible solutions follows the following limits:

$$
\begin{gathered}
P_{G i}^{\min } \leq P_{G i} \leq P_{G i}^{\max }, \\
V_{i}^{\min } \leq V_{i} \leq V_{i}^{\max }, \\
Q_{C i}^{\min } \leq Q_{C i} \leq Q_{C i}^{\max }, \\
T_{i}^{\min } \leq T_{i} \leq T_{i}^{\max },
\end{gathered}
$$

where $P_{G i}$ is the active power generation at bus $i, V_{i}$ is the voltage magnitude at bus $i, Q_{C i}$ is the shunt compensation at bus $i$, and $T_{i}$ is the transformer tap setting at bus $i$. Moreover, $P_{G i}^{\min }$ and $P_{G i}^{\max }$ are lower and upper limits of active power generation, $V_{i}^{\min }$ and $V_{i}^{\max }$ are lower and upper limits of voltage magnitude, $Q_{C i}^{\min }$ and $Q_{C i}^{\max }$ are lower and upper limits of shunt compensation, and $T_{i}^{\mathrm{min}}$ and $T_{i}^{\max }$ are lower and upper limits of tap setting.
5.4. Penalty Function. Penalty function is derived in order to convert constrained problem to unconstrained problem by adding penalty terms. Since RPO problem consists of several constraints as mentioned in the previous subsection, penalty terms have been added to (10) and the equation for the objective function of the problem now becomes

$$
P(x)=P_{\text {loss }}+\Omega_{P}+\Omega_{Q}+\Omega_{V}+\Omega_{G}+\Omega_{C}+\Omega_{T},
$$

where $P(x)$ is the penalty function and $\Omega_{P}, \Omega_{Q}, \Omega_{V}, \Omega_{G}$, $\Omega_{C}$, and $\Omega_{T}$ are the penalty terms of the listed equality and inequality constraints, respectively. Thus, the penalty terms are given by

$$
\begin{aligned}
\Omega_{P}= & \rho \sum_{i=1}^{N}\left\{P_{G i}-P_{D i}-V_{i} \sum_{i=1}^{N} V_{j}\left(g_{i j} \sin \theta_{i j}+B_{i j} \cos \theta_{i j}\right)\right\}^{2}, \\
\Omega_{Q}= & \rho \sum_{i=1}^{N}\left\{Q_{G i}+Q_{C i}-Q_{D i}\right. \\
\Omega_{V}= & \rho \sum_{i=1}^{N}\left\{\max \left(0, V_{i}-V_{i}^{\max }\right)\right\}^{2} \\
& +\rho \sum_{i=1}^{N}\left\{\max \left(0, V_{i}^{\min }-V_{i}\right)\right\}^{2} \\
& \left.+\rho \sum_{i=1}^{N}\left\{\sin \theta_{i j}-B_{i j} \cos \theta_{i j}\right)\right\}^{2}, \\
\Omega_{T}= & \rho \sum_{i=1}^{N G}\left\{\max \left(0, P_{G i}-P_{G i}^{\max }\right)\right\}^{2} \\
& +\rho \sum_{i=1}^{N T}\left\{\max \left(0, Q_{C i}^{\min }-T_{C i}\right)\right\}^{2}, \\
& +\rho \sum_{i=1}^{N G}\left\{\max \left(0, P_{G i}^{\min }-P_{G i}\right)\right\}^{2}, \\
& \rho \sum_{i=1}^{N C}\left\{\max \left(0, Q_{C i}-Q_{C i}^{\max }\right)\right\}^{2} \\
\Omega_{C} &
\end{aligned}
$$

where $P_{G i}$ is the active power generation at bus $i, P_{D i}$ is the active power demand at bus $i, Q_{G i}$ is the reactive power generation at bus $i, Q_{D i}$ is the reactive power demand at bus $i, Q_{C i}$ is the shunt compensation at bus $i, T_{i}$ is the transformer tap settings of transformer $i, B_{i j}$ is the susceptance between bus $i$ and bus $j, N G$ is the total number of generators, $N C$ is the total number of shunt compensator, $N T$ is the total number 
TABLE 3: Performance of optimization algorithms in solving RPO.

\begin{tabular}{lc}
\hline Algorithms & Ploss (MW) \\
\hline SARGA & 4.5740 \\
PSO & 4.6282 \\
CLPSO & 4.5615 \\
EGA-DQLF & 3.2008 \\
ABC & 1.5522 \\
IABC & 1.5185 \\
BABC1 & 1.5215 \\
EABC & 1.5180 \\
JA-ABC5 & $\mathbf{1 . 4 9 8 5}$ \\
\hline
\end{tabular}

of transformers, and the rest of the parameters are the same as in $(10)[31,36]$.

The proposed algorithm, JA-ABC5, is implemented to solve RPO problem by finding the optimal possible solutions to solve the objective function which is the penalty function obtained from (13). The possible solutions that need to be optimized which basically act as the food sources of JA-ABC5 are given by the previous subsection. They are active power generation, $P_{G}$, voltage magnitude, $V$, shunt compensation, $Q_{C}$, and transformer tap setting, $T$, at the required bus. JAABC5 is expected to produce less value of power loss which is affected by the above mentioned control variables' values. Thus, it is important to find the optimal values or settings of the control variables so that less amount of power loss has been generated.

5.5. Results of RPO. For the purpose of solving the RPO problem, IEEE 30-bus power system data has been obtained from [31]. To validate the performance of JA-ABC5 in solving the $\mathrm{RPO}$ problem, it has been compared with three existing $\mathrm{ABC}$ variants: IABC [25], BABC1 [19], and $\mathrm{EABC}[26,29]$ as well as with other optimization algorithms available in the work of [31] which are self-adaptive real coded genetic algorithm (SARGA) [37], particle swarm optimization (PSO) [38], comprehensive learning PSO (CLPSO) [38], and enhanced genetic algorithm with decoupled quadratic load flow (EGADQLF) [39]. The performance of JA-ABC5 in solving the RPO problem in comparison with other optimization algorithms is tabulated in Table 3.

From Table 3, it is clear that variants of ABC algorithm have outperformed the other optimization algorithms. Most importantly, the results have shown that the proposed algorithm, JA-ABC5, has produced the minimum power loss of $1.4985 \mathrm{MW}$ when compared to other optimization algorithms. Thus, this vividly shows that JA-ABC5 is able to solve complex optimization problem and hence can be applied to solve other optimization problems.

\section{Conclusion}

This work presents a new variant of the $\mathrm{ABC}$ algorithm referred to as JA-ABC5 by modifying the standard $\mathrm{ABC}$ algorithm to balance out the effects of exploration and exploitation processes into the performance of the algorithm.
The balanced exploration and exploitation capabilities are able to enhance the performance of the algorithm in terms of convergence speed and global optimum achievement. The performance results have clearly exhibited the best performance of JA-ABC5 in comparison to the compared $\mathrm{ABC}$ variants on 27 benchmark functions. Moreover, the efficiency of the algorithm in solving a complex real-world problem, the reactive power optimization (RPO), has vividly depicted that the algorithm is robust, effective, and reliable in solving optimization problems.

\section{Conflict of Interests}

The authors declare that there is no conflict of interests regarding the publication of this paper.

\section{Acknowledgment}

The authors acknowledge the Ministry of Higher Education (MOHE), Malaysia, FRGS Grant no. 203/PELECT/6071247 for the financial support.

\section{References}

[1] S. Binitha and S. S. Sathya, "A survey of bio inspired optimization algorithms," International Journal of Soft Computing and Engineering, vol. 2, no. 2, pp. 2231-2307, 2012.

[2] Z. Cui, R. Alex, R. Akerkar, and X.-S. Yang, "Recent advances on bioinspired computation," The Scientific World Journal, vol. 2014, Article ID 934890, 3 pages, 2014.

[3] T. Davidović, D. Ramljak, M. Šelmić, and D. Teodorović, "Bee colony optimization for the p-center problem," Computers \& Operations Research, vol. 38, no. 10, pp. 1367-1376, 2011.

[4] A. Q. H. Badar, B. S. Umre, and A. S. Junghare, "Reactive power control using dynamic Particle Swarm Optimization for real power loss minimization," International Journal of Electrical Power \& Energy Systems, vol. 41, no. 1, pp. 133-136, 2012.

[5] N. Karaboga and F. Latifoglu, "Adaptive filtering noisy transcranial Doppler signal by using artificial bee colony algorithm," Engineering Applications of Artificial Intelligence, vol. 26, no. 2, pp. 677-684, 2013.

[6] N. Bacanin and M. Tuba, "Firefly algorithm for cardinality constrained mean-variance portfolio optimization problem with entropy diversity constraint," The Scientific World Journal, vol. 2014, Article ID 721521, 16 pages, 2014.

[7] I. C. Obagbuwa and A. O. Adewumi, "An improved cockroach swarm optimization," The Scientific World Journal, vol. 2014, Article ID 375358, 13 pages, 2014.

[8] Y. Zhou, J. Xie, L. Li, and M. Ma, "Cloud model bat algorithm," The Scientific World Journal, vol. 2014, Article ID 237102, 11 pages, 2014.

[9] A. G. Abro and J. Mohamad-Saleh, "Multiple-global-best guided artificial bee colony algorithm for induction motor parameter estimation," Turkish Journal of Electrical Engineering \& Computer Sciences, vol. 22, no. 3, pp. 620-636, 2014.

[10] K. Charansiriphaisan, S. Chiewchanwattana, and K. Sunat, "A comparative study of improved artificial bee colony algorithms applied to multilevel image thresholding," Mathematical Problems in Engineering, vol. 2013, Article ID 927591, 17 pages, 2013. 
[11] H. A. Abbass, "MBO: marriage in honey bees optimization a haplometrosis polygynous swarming approach," in Proceedings of the Congress on Evolutionary Computation, vol. 1, pp. 207-214, Seoul, Korea, May 2001.

[12] D. Karaboga, "An idea based on honey bee swarm for numerical optimization," Tech. Rep. TR06, 2005.

[13] Y. Marinakis, M. Marinaki, and G. Dounias, "Honey bees mating optimization algorithm for the Euclidean traveling salesman problem," Information Sciences, vol. 181, no. 20, pp. 4684-4698, 2011.

[14] T. Niknam, H. D. Mojarrad, H. Z. Meymand, and B. B. Firouzi, "A new honey bee mating optimization algorithm for nonsmooth economic dispatch," Energy, vol. 36, no. 2, pp. 896-908, 2011.

[15] O. Duangphakdee, S. E. Radloff, C. W. W. Pirk, and H. R. Hepburn, "Waggle dances and azimuthal windows," Psyche, vol. 2011, Article ID 318985, 7 pages, 2011.

[16] D. Karaboga and B. Basturk, "On the performance of artificial bee colony (ABC) algorithm," Applied Soft Computing Journal, vol. 8, no. 1, pp. 687-697, 2008.

[17] M. El-Abd, "Performance assessment of foraging algorithms vs. evolutionary algorithms," Information Sciences, vol. 182, pp. 243-263, 2012.

[18] D. Karaboga and B. Akay, "A comparative study of artificial Bee colony algorithm," Applied Mathematics and Computation, vol. 214, no. 1, pp. 108-132, 2009.

[19] W. Gao, S. Liu, and L. Huang, "A global best artificial bee colony algorithm for global optimization," Journal of Computational and Applied Mathematics, vol. 236, no. 11, pp. 2741-2753, 2012.

[20] W.-F. Gao and S.-Y. Liu, "A modified artificial bee colony algorithm," Computers and Operations Research, vol. 39, no. 3, pp. 687-697, 2012.

[21] A. G. Abro and J. Mohamad-Saleh, "Enhanced probabilityselection artificial bee colony algorithm for economic load dispatch: a comprehensive analysis," Engineering Optimization, vol. 46, no. 10, pp. 1315-1330, 2014.

[22] G. Li, P. Niu, and X. Xiao, "Development and investigation of efficient artificial bee colony algorithm for numerical function optimization," Applied Soft Computing Journal, vol. 12, no. 1, pp. 320-332, 2012.

[23] G. Zhu and S. Kwong, "Gbest-guided artificial bee colony algorithm for numerical function optimization," Applied Mathematics and Computation, vol. 217, no. 7, pp. 3166-3173, 2010.

[24] A. Banharnsakun, T. Achalakul, and B. Sirinaovakul, "The bestso-far selection in Artificial Bee Colony algorithm," Applied Soft Computing Journal, vol. 11, no. 2, pp. 2888-2901, 2011.

[25] W. Gao and S. Liu, "Improved artificial bee colony algorithm for global optimization," Information Processing Letters, vol. 111, no. 17, pp. 871-882, 2011.

[26] A. G. Abro and J. Mohamad-Saleh, "Enhanced global-best artificial bee colony optimization algorithm," in Proceedings of the 6th UKSim-AMSS European Modelling Symposium (EMS '12), pp. 95-100, Valetta, Malta, November 2012.

[27] A. G. Abro and J. Mohamad-Saleh, "An enhanced artificial bee colony optimization algorithm," in Recent Advances in Systems Science and Mathematical Modelling, WSEAS Press, 2012.

[28] N. Sulaiman, J. Mohamad-Saleh, and A. G. Abro, "A modified artificial bee colony (JA-ABC) optimization algorithm," in Proceedings of the International Conference on Applied Mathematics and Computational Methods in Engineering, pp. 74-79, Rhodes Island, Greece, July 2013.
[29] A. G. Abro, Performance enhancement of artificial bee colony optimization algorithm [Ph.D. thesis], 2013.

[30] D. Karaboga and B. Basturk, "A powerful and efficient algorithm for numerical function optimization: artificial bee colony (ABC) algorithm," Journal of Global Optimization, vol. 39, no. 3, pp. 459-471, 2007.

[31] K. Ayan and U. K1lıç, "Artificial bee colony algorithm solution for optimal reactive power flow," Applied Soft Computing, vol. 12, no. 5, pp. 1477-1482, 2012.

[32] J. Zhu, Optimization of Power System Operation, John Wiley \& Sons, New York, NY, USA, 2009.

[33] J. Rahul, Y. Sharma, and D. Birla, "A new attempt to optimize optimal power flow based transmission losses using genetic algorithm," in Proceedings of the 4th International Conference on Computational Intelligence and Communication Networks (CICN '12), pp. 566-570, Mathura, India, November 2012.

[34] C. Sumpavakup, I. Srikun, and S. Chusanapiputt, "A solution to the optimal power flow using artificial bee colony algorithm," in Proceedings of the International Conference on Power System Technology (POWERCON '10), pp. 1-5, Hangzhou, China, October 2010 .

[35] A. M. Abusorrah, "Optimal power flow using adaptive fuzzy logic controllers," Mathematical Problems in Engineering, vol. 2013, Article ID 975170, 7 pages, 2013.

[36] U. Leeton, D. Uthitsunthorn, U. Kwannetr, N. Sinsuphun, and T. Kulworawanichpong, "Power loss minimization using optimal power flow based on particle swarm optimization," in Proceedings of the 7th Annual International Conference on Electrical Engineering/Electronics, Computer, Telecommunications and Information Technology (ECTI-CON '10), pp. 440-444, Chiang Mai, Thailand, May 2010.

[37] P. Subbaraj and P. N. Rajnarayanan, "Optimal reactive power dispatch using self-adaptive real coded genetic algorithm," Electric Power Systems Research, vol. 79, no. 2, pp. 374-381, 2009.

[38] K. Mahadevan and P. S. Kannan, "Comprehensive learning particle swarm optimization for reactive power dispatch," Applied Soft Computing Journal, vol. 10, no. 2, pp. 641-652, 2010.

[39] M. S. Kumari and S. Maheswarapu, "Enhanced genetic algorithm based computation technique for multi-objective optimal power flow solution," International Journal of Electrical Power \& Energy Systems, vol. 32, no. 6, pp. 736-742, 2010. 

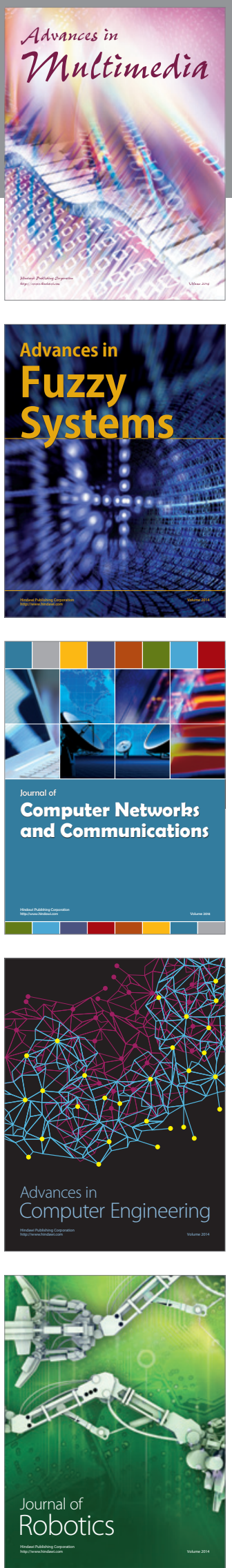

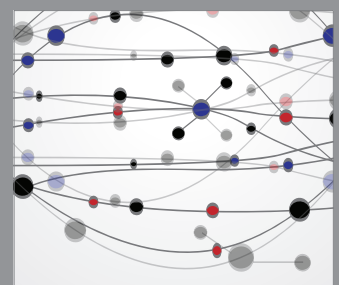

The Scientific World Journal
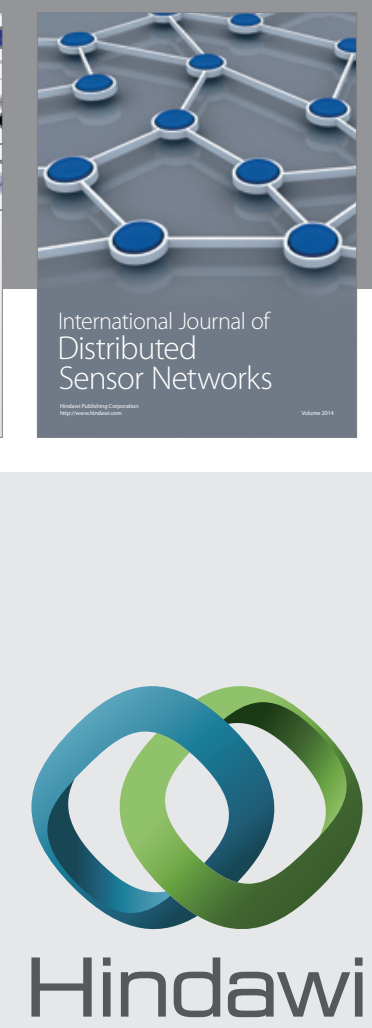

Submit your manuscripts at

http://www.hindawi.com
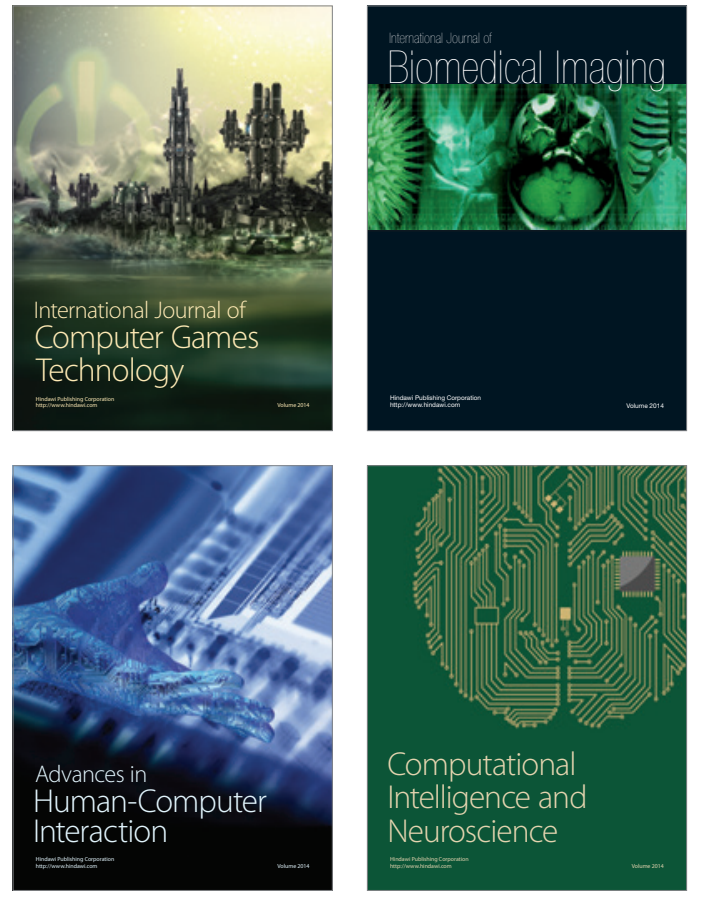
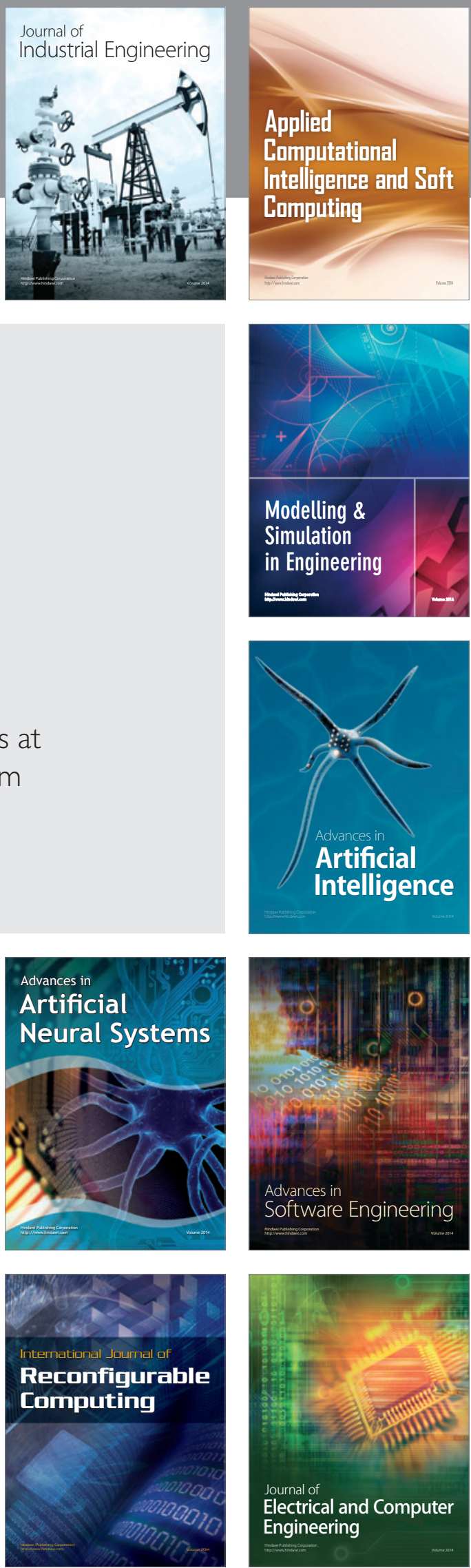